\title{
Molecular Characterization, Nutritional and Insulin Regulation of Elovl6 in Rainbow Trout (Oncorhynchus mykiss)
}

\author{
Yongnan Li ${ }^{1}$, Yuning Pang ${ }^{1}$, Zengqi Zhao ${ }^{1}$, Xiaojun Xiang ${ }^{1}$, Kangsen Mai ${ }^{1,2}$ and \\ Qinghui Ai ${ }^{1,2, *}$ \\ 1 Key Laboratory of Aquaculture Nutrition and Feed (Ministry of Agriculture) \& Key Laboratory of \\ Mariculture (Ministry of Education), Ocean University of China, 5 Yushan Road, Qingdao 266003, China; \\ nanyongli2013@163.com (Y.L.); 15530793072@163.com (Y.P.); Zhaozengqi123456@163.com (Z.Z.); \\ xxj_ouc@163.com (X.X.); kmai@ouc.edu.cn (K.M.) \\ 2 Laboratory for Marine Fisheries Science and Food Production Processes, Qingdao National Laboratory for \\ Marine Science and Technology, 1 Wenhai Road, Qingdao 266237, China \\ * Correspondence: qhai@ouc.edu.cn; Tel.: +86-0532-82031943
}

Received: 14 December 2019; Accepted: 29 January 2020; Published: 10 February 2020

\begin{abstract}
Elongation of very long-chain fatty acids protein 6 (Elovl6) is a crucial enzyme in the synthesis of endogenous fatty acids, which participates in the energy balance and metabolic diseases. The main objective of this study was to explore the molecular characterization of Elovl6 and the regulation of elovl6 expression in response to dietary fatty acids and insulin. In the present study, the ORF (open reading frame) of Elovl6 from rainbow trout was cloned and characterized, which showed a high identity $(87 \%)$ with mammals and other teleost. The results of quantitative PCR showed that the transcriptional levels of elovl6 from rainbow trout that were fed diets containing soybean oil (enriched with 18:2n-6, linoleic acid (LA)) or linseed oil (enriched with 18:3n-3, $\alpha$-linolenic acid (ALA)) were lower than those in the group that were fed diets containing fish oil (enriched with 20:5n-3, eicosapentaenoic acid (EPA) and 22:6n-3, docosahexaenoic acid (DHA)). Correspondingly, mRNA expression of elovl6 in hepatocytes treated with DHA was dramatically higher than that in LA and ALA groups. The transcriptional expression of elovl6 in hepatocytes treated with insulin was also significantly increased. Moreover, the dual luciferase assay showed the transcription factor CREB1 dramatically up-regulated the promoter activity of elovl6, while FOXO1 significantly down-regulated the elovl6 promoter activity in rainbow trout. The differences in transcriptional expression of $c r b e 1$ and foxo 1 may contribute to the increase or decrease of elovl6 expression in rainbow trout in response to fatty acids or insulin. These findings revealed the molecular characterization of elovl 6 and the regulation of elovl6 expression by CREB1 and FOXO1 in rainbow trout in response to dietary fatty acids or insulin.
\end{abstract}

Keywords: rainbow trout; elovl6; fatty acids; insulin

\section{Introduction}

De novo lipogenesis (DNL) is a process of converting excess carbohydrates into fatty acids and triglycerides, which is an essential and complex physiological process [1]. As the unique and last elongase involved in the synthesis of endogenous fatty acids in DNL, elongation of very long-chain fatty acids protein 6 (Elovl6) acted to catalyze the elongation of palmitate (PA, C16:0) or palmitoleic acid (POA, C16:1n-7) to stearate (C18:0) or vaccenic acid (C18:1n-7) [2,3]. Recent studies have suggested that Elovl6 is related to energy balance and metabolic diseases, which could be regulated by nutrients and hormones [4]. 
Elovl6 was highly expressed in the liver, brain, and adipose tissue, which played a crucial role in maintaining the metabolic balance of the fatty acids [5]. Loss of Elovl6 function reduced the concentrations of $\mathrm{C} 18: 0$ and $\mathrm{C} 18: 1 \mathrm{n}-7$, but increased $\mathrm{C} 16: 0$ and $\mathrm{C} 16: 1 \mathrm{n}-7$ contents in mice (Mus musculus) [6], and dietary fatty acids (PUFAs) could profoundly regulate the expression of elovl6 in mice [5]. In the large yellow croaker (Larimichthys crocea), elovl6 expression was increased with the replacement of fish oil by soybean oil or linseed oil in diets via the regulation of transcription factors including hepatocyte nuclear factor $1 \alpha(\mathrm{HNF} 1 \alpha)$, CCAAT-enhancer-binding protein $\beta(\mathrm{CEBP} \beta)$, retinoid $X$ receptor $\alpha(R X R \alpha)$, and cAMP response element-binding protein (CREB1) [7].

Furthermore, Elovl6 also showed a crucial function for insulin resistance [3]. The deletion of elovl6 in mice could prevent diet-induced insulin resistance, and specific deletion of elovl6 in leptin receptor-deficient mice increased insulin adaptability [3,8]. However, in aquatic animals, Elovl6 was only cloned in Misgurnus anguillicaudatus [9], Eriocheir sinensis [10], and Larimichthys crocea [7] to date, the regulation of dietary fatty acids or insulin on elovl6 expression is still poorly understood.

Rainbow trout (Oncorhynchus mykiss) is an essential commercial freshwater fish that has been widely farmed around the world. The objective of the present study was to investigate the molecular characterization of elovl6, and the regulatory mechanism of elovl6 expression in response to dietary fatty acids or insulin in rainbow trout. The results may strengthen the understanding of nutritional and hormonal regulation, and add new data about the regulatory mechanism of the metabolism in rainbow trout, which could aid in the healthy and sustainable wide aquaculture of rainbow trout.

\section{Materials and Methods}

\subsection{Animal Experiments}

The present study was carried out in strict accordance with the Management Rule of Laboratory Animals (Chinese Order No. 676 of the State Council, revised 1 March 2017). With fish oil, soybean oil, and linseed oil as the lipid source, three isoproteic and isolipidic diets (43\% crude protein and $12 \%$ crude fat) were prepared and labeled as fish oil (FO), soybean oil (SO), and linseed oil (LO). Fish fasted for $24 \mathrm{~h}$ before the experiment. The fish with similar size (mean weight $10.03 \pm 0.02 \mathrm{~g}$ ) were obtained from a commercial farm in Weifang, Shandong, China, and randomly assigned to nine tanks in groups of 30 fish each with continuous flow-through freshwater supply and aeration, with water temperature maintained at $18 \pm 2{ }^{\circ} \mathrm{C}$ and dissolved oxygen at 7-8 mg/L. Triplicate groups of fish were randomly allocated to $\mathrm{FO}, \mathrm{SO}$, and $\mathrm{LO}$, respectively. The fish were fed with each diet to satiation by hand twice daily at 06:00 and 18:00, respectively, for ten weeks. At the end of the experiment, liver, muscle, intestine, heart, spleen, gill, eye, brain, and adipose tissue were collected for RNA isolation and gene expression analyses after fish fasted for $24 \mathrm{~h}$.

\subsection{RNA Isolation and $c D N A$ Synthesis}

Total RNA extraction and cDNA synthesis were performed by TransZol (TransGen Biotech, Beijing, China) and cDNA by the PrimeScriptTM RT reagent kit (Takara, Japan) according to the manufacturer's instructions.

\subsection{Cloning, Sequence, and Phylogenetic Analysis of Elovl6}

The cloning primers of elovl6 were designed based on the sequence of rainbow trout on NCBI (XM_021584104.1) (Table 1). Primers were synthesized by Sangon Biotech Co., Ltd., Shanghai, China. PCR was performed in PrimeSTAR ${ }^{\circledR}$ Max DNA Polymerase (Takara, Japan) and the total system was $25 \mu \mathrm{L}\left(1 \mu \mathrm{L}\right.$ liver cDNA, $1 \mu \mathrm{L}$ each primer, $12.5 \mu \mathrm{L}$ PrimeSTAR ${ }^{\circledR} \mathrm{Max}$, and $9.5 \mu \mathrm{L}$ DEPC water). PCR amplification was carried out for 35 cycles under the following procedure: $98{ }^{\circ} \mathrm{C}$ for $10 \mathrm{~s}, 55{ }^{\circ} \mathrm{C}$ for $15 \mathrm{~s}$, and $72{ }^{\circ} \mathrm{C}$ for $1 \mathrm{~min}$, followed by $72{ }^{\circ} \mathrm{C}$ for $10 \mathrm{~min}$. The PCR products were separated by electrophoresis on a $1.0 \%$ agarose gel, purified and ligated into the pEASY-T1 vector (TransGen, Beijing, China) for further sequencing (Sangon Biotech Co., Ltd., Shanghai, 
China). Both the nucleotide sequence and the amino acid sequence of Elovl6 were analyzed on the NCBI blast program (https://blast.ncbi.nlm.nih.gov/Blast.cgi). The multiple sequence alignment was performed by DNAMAN (Lynnon BioSoft, Vaudreuil, Quebec, Canada) and ESPript 3.0 (http: //espript.ibcp.fr/ESPript/cgi-bin/ESPript.cgi). The phylogenetic analyses were constructed by the MEGA6 program and the tree was obtained by the neighbor-joining method.

Table 1. Sequences of the primers for gene cloning, qPCR analysis, and promoter cloning.

\begin{tabular}{|c|c|c|}
\hline Primer & Sequences $5^{\prime}-3^{\prime}$ & Primer Information \\
\hline ORF-F & ATGTCGGTGTTGGCTCTGCAAG & Elovl6-ORF \\
\hline ORF-R & CTACTGGGTCTTCCTTGCGGC & Elovl6-ORF \\
\hline qElovl6-F & TCAACGAGGACGAAGCCATACGA & Elov16 q-PCR \\
\hline qElovl6-R & CCCAGTGCGGACAGCACCAAATA & Elov16 q-PCR \\
\hline qCREB1-F & AGGAGTCAGTGGACAGTGTGA & CREB1 q-PCR \\
\hline qCREB1-R & TGCTGGTCTGGTAGATAGGGC & CREB1 q-PCR \\
\hline qFOXO1-F & AACTCCCACAGCCACAGCAA & FOXO1 q-PCR \\
\hline qFOXO1-R & CGATGTCCTGTTCCAGGAAGG & FOXO1 q-PCR \\
\hline$\beta$-actin-F & ATCAGGGAGTGATGGTTGGGATG & $\beta$-actin q-PCR \\
\hline$\beta$-actin-R & CTCGTAGATGGGTACTGTGTGGG & $\beta$-actin q-PCR \\
\hline E6-P-F & GTAAACTGTTGCTGGAGATTCCGGAC & Elovl6 promoter \\
\hline E6-P-R & GTTCACTGTGCGCTTTCCTGTAAACG & Elovl6 promoter \\
\hline $\mathrm{PCS}^{+}{ }^{+}$SREBP1-F & ATGAACTTGTCTTTTGACGATCAG & Expression plasmid \\
\hline $\mathrm{PCS}^{+}{ }^{+}$SREBP1-R & CTAGGCAGAGGTGACAGTGGTGC & Expression plasmid \\
\hline $\mathrm{PCS}^{+}{ }^{+}$-SREBP2-F & ATGGACAGTAACGTTAGTGGGGAG & Expression plasmid \\
\hline $\mathrm{PCS}^{+}{ }^{+}$-SREBP2-R & TCAGGAGGCCGCGATGGTG & Expression plasmid \\
\hline $\mathrm{PCS} 2^{+}-\mathrm{CEBP} \alpha-\mathrm{F}$ & ATGGAGCAACCAAACCTCTACGAG & Expression plasmid \\
\hline $\mathrm{PCS}^{+}-\mathrm{CEBP} \alpha-\mathrm{R}$ & TCACTGGCAGTTGGCCAATG & Expression plasmid \\
\hline $\mathrm{PCS} 2^{+}-\mathrm{CEBP} \beta-\mathrm{F}$ & TGGAAGTGGCCGGTTTCTACG & Expression plasmid \\
\hline $\mathrm{PCS}^{+}{ }^{+} \mathrm{CEBP} \beta-\mathrm{R}$ & CTAACCGGTGGCAGAAAGCAAG & Expression plasmid \\
\hline $\mathrm{PCS}^{+}-\mathrm{HNF} 1 \alpha-\mathrm{F}$ & ATGGAGGGAGAGGAGAGGAAAGG & Expression plasmid \\
\hline $\mathrm{PCS}^{+}-\mathrm{HNF} 1 \alpha-\mathrm{R}$ & CTACTGTGCGGTAGAGACCATCTGT & Expression plasmid \\
\hline $\mathrm{PCS}^{+}-\mathrm{PPAR} \gamma-\mathrm{F}$ & ATGCATATGATGTGTAGCAATTTTA & Expression plasmid \\
\hline $\mathrm{PCS}^{+}-\mathrm{PPAR} \gamma-\mathrm{R}$ & CTAGTAGAGGTCTCTCATGATCTCCT & Expression plasmid \\
\hline $\mathrm{PCS}^{+}-\mathrm{RXR} \alpha-\mathrm{F}$ & ATGACGCTGGAAATTCTGACATATT & Expression plasmid \\
\hline $\mathrm{PCS}^{+}-\mathrm{RXR} \alpha-\mathrm{R}$ & TTATGTCATTTGGTGGGGCG & Expression plasmid \\
\hline $\mathrm{PCS}^{+}{ }^{-} \mathrm{CREB} 1-\mathrm{F}$ & ATGACCATGGAGTCGGGAGC & Expression plasmid \\
\hline $\mathrm{PCS}^{+}+\mathrm{CREB} 1-\mathrm{R}$ & CTACTCAGATTTATGGCAGTACAGGTC & Expression plasmid \\
\hline $\mathrm{PCS}^{+}-\mathrm{P} 65-\mathrm{F}$ & ATGGATGGAATGTATGGATGGGG & Expression plasmid \\
\hline $\mathrm{PCS}^{+}-\mathrm{P} 65-\mathrm{R}$ & TAAGTCTGATGTCCGGACACGAA & Expression plasmid \\
\hline $\mathrm{PCS}^{+}-\mathrm{FOXO} 1-\mathrm{F}$ & ATGGCAGAATTACCACCGCCG & Expression plasmid \\
\hline $\mathrm{PCS}^{+}-\mathrm{FOXO} 1-\mathrm{R}$ & CTAGCCAGACACCCAGCTGTGTGTG & Expression plasmid \\
\hline
\end{tabular}

\subsection{Real-Time Quantitative PCR (RT-qPCR) Analysis}

qPCR of the target genes including elovl6, forkhead transcription factor (foxo1), and creb1 in rainbow trout was performed in a quantitative thermal cycler (CFX96TM Real-Time System, BIO-RAD, Hercules, CA, USA) by a SYBR Green real-time PCR kit (Takara, Japan). The primers for RT-qPCR were designed by Primer Premier 5.0 (Premier Biosoft, San Francisco, CA, USA) based on the cloned nucleotide sequences, which are listed in Table 1 . The results of real-time quantitative experiments were normalized to that of $\beta$-actin by the $2^{-\Delta \Delta C t}$ method [11].

\subsection{Cloning of Elovl6 Promoter from Rainbow Trout}

The genomic DNA of rainbow trout was isolated by the FastPure Cell/Tissue DNA Isolation Mini Kit (Vazyme, Nanjing, China) according to the manufacturer's instructions. The promoter sequence of elovl6 was cloned by genomic DNA and inserted into the pGL3-basic luciferase reporter plasmid (Promega, Madison, WI, USA). The plasmid was extracted by the Easypure ${ }^{\circledR}$ Hipure Plasmid MiniPrep 
Kit (TransGen, Beijing, China). JASPAR (http://jaspar.genereg.net/) and TF binding (http://tfbind.hgc.jp/) were used to predict potential transcription factor binding sites in the promoter of elovl6 in rainbow trout. We set up the relative profile score threshold as $80 \%$ and selected the transcription factors with high scores and conservative binding sites to study in the present study.

\subsection{Construction of Transcription Factor Plasmids, Cell Culturing, Transfection, and Luciferase Assay}

For transcription factor plasmids, the ORFs (open reading frames) of sterol regulatory element-binding protein 1 (SREBP1, XM_021624594.1), sterol regulatory element-binding protein 2 (SREBP2, XM_021625095.1), HNF1 $\alpha$ (XM_021621309.1), CCAAT-enhancer-binding protein $\alpha(C E B P \alpha$, NM_001172496.1), CEBP $\beta$ (NM_001124447.1), CREB1 (XM_021597386.1), FOXO1 (XM_021618954.1), RXR $\alpha$ (XM_021590263.1), P65 (XM_021578194.1), and peroxisome proliferator-activated receptor $\gamma$ (PPAR $\gamma$, XM_021610844.1) were cloned into PCS2 ${ }^{+}$plasmids (Invitrogen, Carlsbad, CA, USA) by the ClonExpress II One Step Cloning Kit (Vazyme, Nanjing, China).

HEK 293T cells were cultured according to the method described in the previous studies [12]. Transfection was performed using Lipofectamine 3000 (Invitrogen, Carlsbad, CA, USA) according to the instructions. A total of $200 \mathrm{ng}$ of promoter reporter plasmid, $600 \mathrm{ng}$ of transcription factor plasmid, $20 \mathrm{ng}$ of pRL-TK renilla luciferase, and $2.0 \mu \mathrm{L}$ Lipofectamine 3000 were co-transfected in the 24-well plate in triplicate wells with three independent experiments. The TransDetect Double-Luciferase Reporter Assay Kit (TransGen, Beijing, China) and InfiniTE200 plate reader (Tecan, Männedorf, Switzerland) were used for the detection of dual luciferase activity.

\subsection{Primary Hepatocyte Isolation and Incubation}

The primary hepatocyte of rainbow trout was isolated by the perfusion method. Rainbow trout fasted for $24 \mathrm{~h}$ before the experiment. After anesthesia with MS-222, the fish was dissected to expose the liver tissue. Hanks (Solarbio, Beijing, China) was injected into the hepatic portal vein until the liver became utterly white. Then, collagenase was pushed slowly into the liver for $30 \mathrm{~min}$. The liver was cut into small fragments, centrifuged at $700 \times g$ for $5 \mathrm{~min}$ after filtration, and the obtained cells were washed with complete medium (DMEM-F12 + 15\% FBS). Then the cells were diluted to a certain concentration and stored at $18{ }^{\circ} \mathrm{C}$. Fatty acids, including linoleic acid (LA), $\alpha$-linolenic acid (ALA), eicosapentaenoic acid (EPA), and docosahexaenoic acid (DHA) (Cayman Chemical Co., Ann Arbor, MI, USA), were supplemented in the form of BSA/fatty acid complexes at $100 \mu \mathrm{M}$ for $12 \mathrm{~h}$ in triplicate wells with three independent experiments. Furthermore, the primary hepatocyte of rainbow trout was treated with insulin ( $1 \mathrm{nM}, 10 \mathrm{nM}$, and $100 \mathrm{nM}$, Solarbio, Beijing, China) for $6 \mathrm{~h}$ and $8 \mathrm{~h}$ in triplicate wells with three independent experiments. The concentrations and incubation times of fatty acids and insulin were determined by a preliminary experiment. After incubation, cells were lysed in the wells and harvested for RNA extraction.

\subsection{Statistical Analysis}

Statistical analysis of all data was performed using SPSS 20.0 and the results were presented as means \pm S.E.M. One-way ANOVA and Tukey's test were used to inspect the differences among more than two groups, and the differences between two groups were determined by Student's $t$ test.

\section{Results}

\subsection{Molecular Characterization of Rainbow Trout Elovl6}

The rainbow trout elovl6 cDNA contained an ORF of 828 bp encoding a putative protein of 275 amino acids (AA) (Figure 1). BLAST analysis of an amino acid sequence indicated that Elovl6 in rainbow trout shared high sequence identity with Oncorhynchus kisutch (99\%), Salmo salar (97\%), Larimichthys crocea (93\%), Labrus bergylta (91\%), Cyprinus carpio (91\%), and Danio rerio (90\%). 


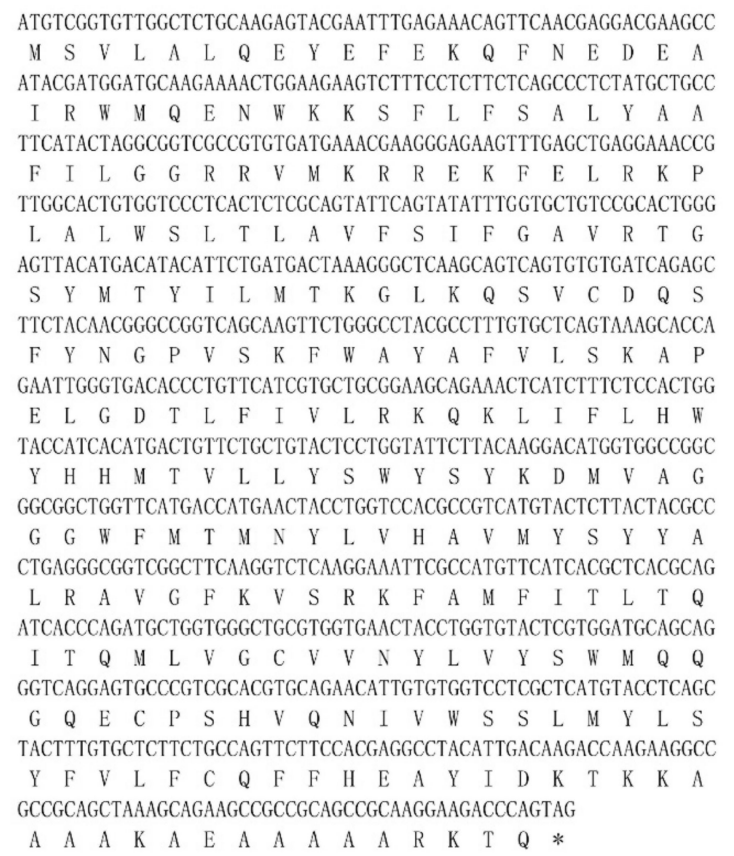

Figure 1. Nucleotide and deduced amino acid sequences of the elongation of very long-chain fatty acids protein 6 (Elovl6) in rainbow trout. ${ }^{*}$ Represents the stop codon.

Rainbow trout Elovl6 protein possessed the characteristic features of Elovl family members, including histidine box (HXXHH) and six membrane-spanning domains (Figure 2). The deduced amino acid sequences showed an identity of $87 \%$ with the six species. Phylogenetic analysis clustered rainbow trout Elovl6 with other teleosts and mammals (Figure 3).

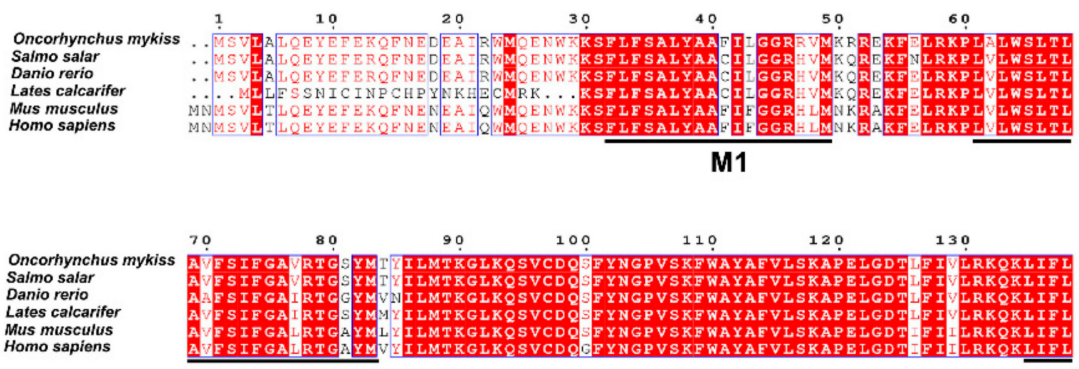

M2
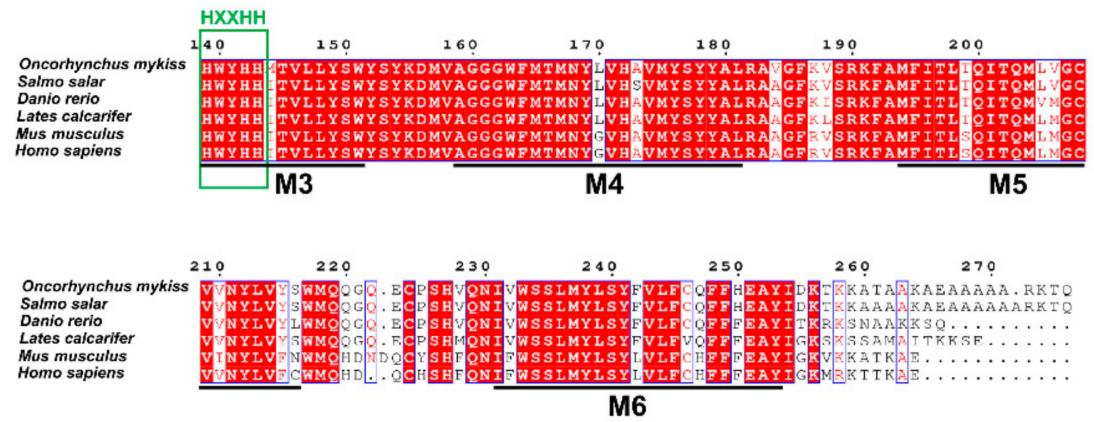

Figure 2. Amino acid alignment of Elovl6 amino acid sequence in rainbow trout with other species. The conserved HXXHH histidine box motif (green box) and putative membrane-spanning domains (M1-M6) are indicated. The blue boxes represent the conserved sequence. The identity shading is based on $87 \%$ identity threshold. 


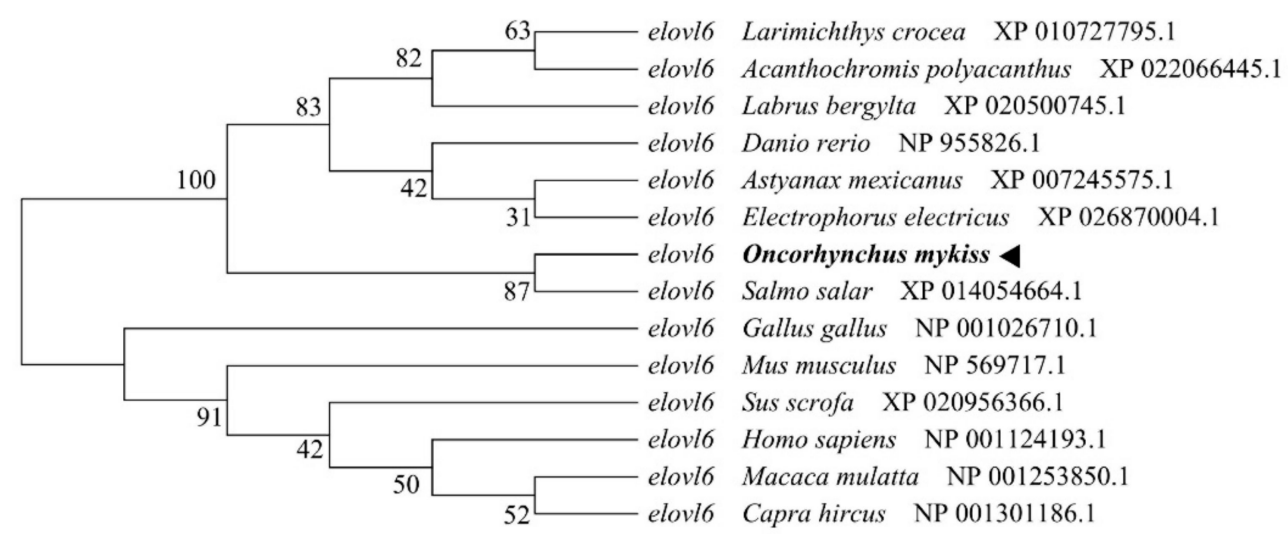

Figure 3. Phylogenetic tree of elvol6 in rainbow trout and other vertebrate counterparts. The neighbor joining method in MEGA6 was used to construct the tree. The numbers represent the frequencies (\%) with which the tree topology presented was replicated after 1000 iterations.

\subsection{Tissue Distribution of Rainbow Trout Elovl6}

The transcriptional expression of elovl 6 was determined in different tissues of rainbow trout, such as the brain, spleen, intestine, gill, liver, muscle, eye, heart, and adipose tissues (Figure 4). The high expression of elovl6 in rainbow trout was observed in the brain, liver, and eye. Furthermore, the highest and lowest expression was detected in the brain and spleen, respectively.

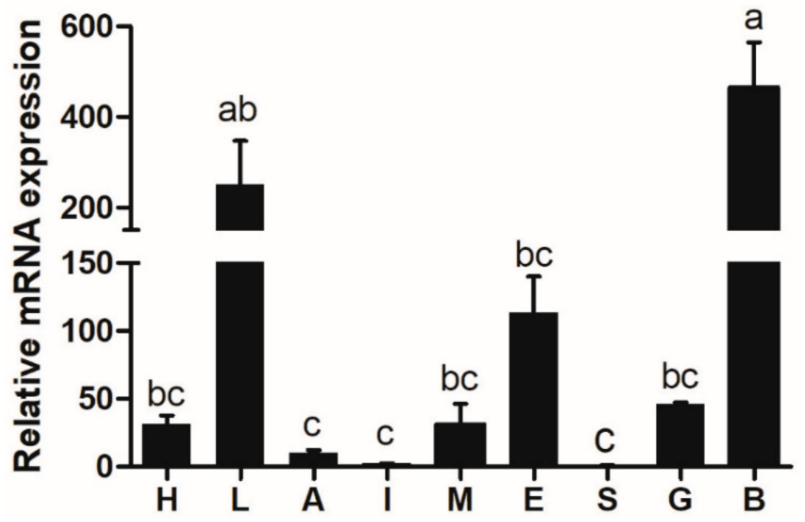

Figure 4. The transcriptional expression of elovl6 in different tissues of rainbow trout. Heart $(\mathrm{H})$, liver $(\mathrm{L})$, adipose tissue (A), intestine (I), muscle (M), eye (E), spleen (S), gill (G), brain (B). The expression of elvol6 in the spleen was selected as normalization. Data were presented as means \pm S.E.M. $(\mathrm{n}=3)$. Means that share the same superscript letter are not significantly different $(p>0.05)$.

\subsection{Transcriptional Expression of Elovl6 in Response to Fatty Acids}

\subsubsection{Expression of Elovl6 in Response to Dietary Fatty Acids}

The transcriptional expression of elovl6 in the liver of rainbow trout that were fed diets containing fish oil (FO), soybean oil (SO), or linseed oil (LO) was determined in the present study (Figure 5A). The expression of the elovl6 gene in rainbow trout that were fed diets containing LO was significantly lower than that in rainbow trout that were fed diets containing FO $(p<0.05)$, and the expression in the $\mathrm{SO}$ group was lower than that in the FO group, but not significantly different $(p>0.05)$. 
A

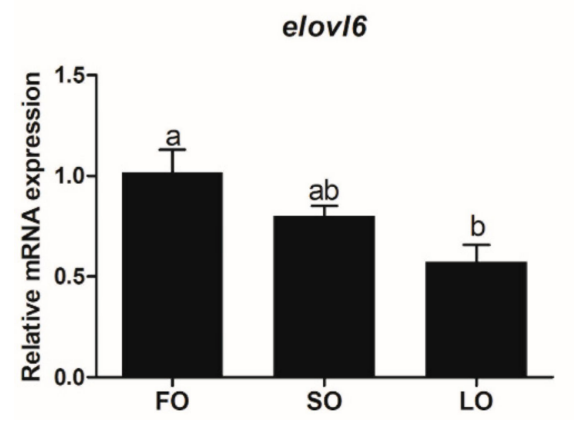

B

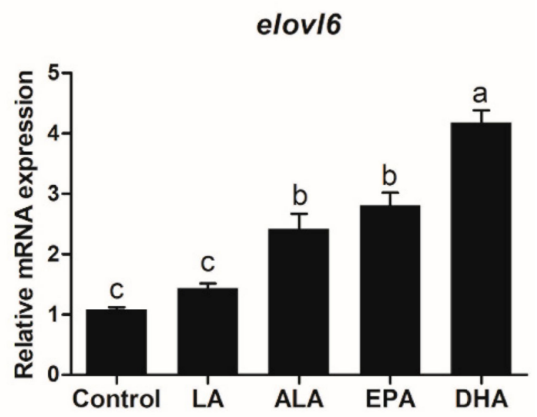

Figure 5. Effects of fatty acids on the transcriptional expression of elovl6 in rainbow trout. (A) The elovl6 expression in the liver of rainbow trout that were fed diets containing fish oil (FO), soybean oil (SO), and linseed oil (LO). (B) The expression of elvol6 in hepatocytes of rainbow trout treated with $100 \mu \mathrm{M}$ linoleic acid (LA), $\alpha$-linolenic acid (ALA), eicosapentaenoic acid (EPA), and docosahexaenoic acid (DHA) for $12 \mathrm{~h}$. FO and the control group were selected as normalization. Data were presented as means \pm S.E.M. $(n=3)$. Means that share the same superscript letter are not significantly different $(p>0.05)$.

\subsubsection{Expression of Elovl6 in Hepatocytes in Response to Fatty Acids}

Incubation of hepatocytes from rainbow trout with fatty acids caused differences in elovl 6 expression (Figure 5B). Compared with the control group, the transcriptional expression of elovl6 in hepatocytes treated with $100 \mu \mathrm{M} \alpha$-linolenic acid (ALA), eicosapentaenoic acid (EPA), or docosahexaenoic acid (DHA) for $12 \mathrm{~h}$ was significantly increased $(p<0.05)$. The expression of elovl6 in hepatocytes treated with $100 \mu \mathrm{M}$ linoleic acid (LA) for $12 \mathrm{~h}$ was the lowest, while the highest expression of elovl6 in hepatocytes was observed in the DHA group.

\subsection{Transcriptional Expression of Elovl6 in Hepatocytes in Response to Insulin}

To study the relationship between Elovl6 and insulin, the transcriptional expression of elovl6 in hepatocytes in response to insulin was determined by qPCR. Compared with the control group, the expression of elovl 6 in hepatocytes of rainbow trout treated with 10 and $100 \mathrm{nM}$ insulin for 6 or $8 \mathrm{~h}$ was significantly increased $(p<0.05)$, and the expression of elovl6 in hepatocytes treated with 10 and $100 \mathrm{nM}$ insulin for $6 \mathrm{~h}$ was significantly higher than that in the $8 \mathrm{~h}$ group $(p<0.01)$ (Figure 6).

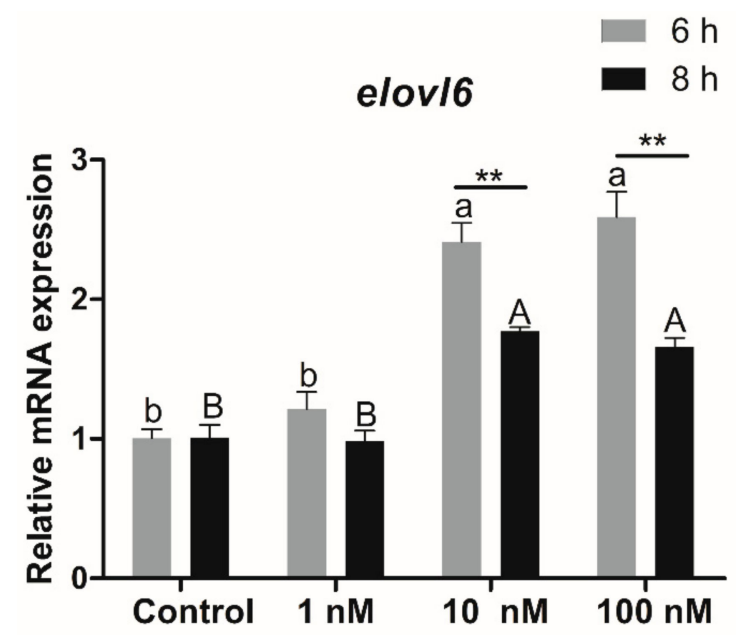

Figure 6. The transcriptional expression of elovl6 in hepatocytes of rainbow trout treated with graded amounts of insulin. Control group was selected as normalization. Data were presented as means \pm S.E.M. $(n=3)$. Capital letters show the differences in the $8 \mathrm{~h}$ group. Lowercase letters show the differences in the $6 \mathrm{~h}$ group. Means that share the same superscript letter are not significantly different $(p>0.05) .{ }^{* *} p<0.01$. 


\subsection{Transcriptional Regulation of the Elovl6 in Response to Fatty Acids or Insulin}

\subsubsection{Regulation of the Elovl6 by Transcription Factors}

The upstream sequence of $2615 \mathrm{bp}$ adjacent to the start of elovl6 ORF was cloned as the promoter of elovl6 in rainbow trout. The binding sites of transcription factors including CEBP $\beta, \operatorname{CEBP} \alpha, H N F 1 \alpha$, PPAR $\gamma$, RXR $\alpha$, SREBP1, SREBP2, CREB1, P65, and FOXO1 were predicted within the elovl 6 promoter region of rainbow trout by bioinformatics software (Figure 7A and Table 2). The dual-luciferase reporter assays in HEK 293T cells showed that the transcription factor CREB1 showed the largest activation of elovl6 promoter activity (7.28-fold), and CEBP $\alpha$, P65, SREBP1, and SREBP2 up-regulated the promoter activity of elovl6 by 2.28-fold, 3.05-fold, 2.08-fold, and 2.52-fold, respectively (Figure 7B). Furthermore, the transcription factor FOXO1 significantly down-regulated the promoter activity of elovl6. However, the transcription factors CEBP $\beta, H N F 1 \alpha, R X R \alpha$, and PPAR $\gamma$ showed no significant regulatory effect on the elovl6 promoter of rainbow trout (Figure 7B).

A

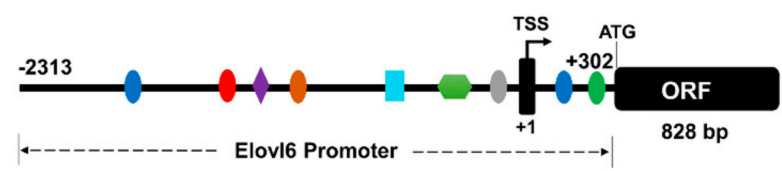

CEBP $\alpha / C E B P \beta$

RXRa FOX01 CREB1

$\checkmark$ P65 HNF1a PPARY

B

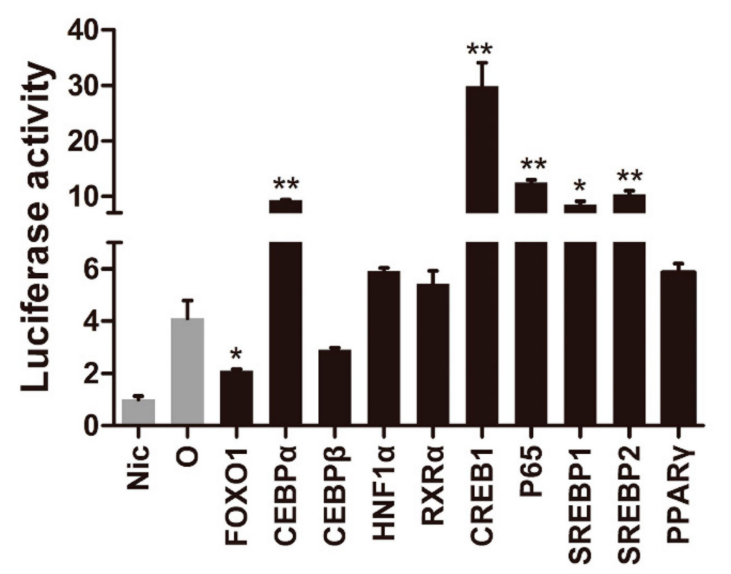

Figure 7. The transcription factors sites (A) and effects on the elovl6 promoter (B) of rainbow trout in HEK 293T cells. The negative control Nic (pGL3-basic) was an empty plasmid with no promoter sequence. TSS: transcriptional start site. The promoter was co-transfected with plasmids of transcription factors FOXO1; CEBP $\alpha$; CEBP $\beta$; HNF1 $\alpha$; RXR $\alpha$; CREB1; P65; SREBP1; SREBP2; and PPAR $\gamma$, compared with the control group $(\mathrm{O})$ which transfected with pGL3-elovl6 promoter only, respectively. The luciferase activity in the Nic group was selected as normalization. Data were presented as means \pm S.E.M. $(n=3)$. ${ }^{*} p<0.05 ;{ }^{* *} p<0.01$. 
Table 2. The transcription factor binding sites predicted by software online.

\begin{tabular}{|c|c|c|c|}
\hline Transcription Factor & Software & Position & Predicted Site \\
\hline \multirow{2}{*}{ СЕВР $\alpha / C Е B P \beta$} & \multirow{2}{*}{ JASPAR } & -1417 & TATTGCACCATA \\
\hline & & +52 & AATTGCAAAATA \\
\hline $\mathrm{RXR} \alpha$ & JASPAR & -1155 & TAACATTAAATAACTTTGG \\
\hline P65 & TF binding & -1081 & GGAAACTCTC \\
\hline SREBP1/SREBP2 & JASPAR & -935 & ATGGGGAGAT \\
\hline FOXO1 & JASPAR & -531 & TGTAAACAAGA \\
\hline HNF1 $\alpha$ & JASPAR & -246 & GGGTAATTGTTTAC \\
\hline CREB1 & JASPAR & -35 & GGACGTCA \\
\hline $\operatorname{PPAR} \gamma$ & JASPAR & +104 & TTGGTGGAGAGGGCC \\
\hline
\end{tabular}

\subsubsection{Transcriptional Expression of Creb1 and Foxo1 in Response to Dietary Fatty Acids}

The transcriptional expression of $c r e b 1$ in the liver of rainbow trout that were fed diets containing $\mathrm{SO}$ and $\mathrm{LO}$ was lower than that in rainbow trout that were fed diets containing $\mathrm{FO}$, and a significant difference was observed between the FO and LO groups $(p<0.05)$ (Figure 8A). The expression of creb1 in the SO group was higher than that in the LO group, but there was no significant difference between the two groups $(p>0.05)$. The expression of the foxo1 gene in the liver of rainbow trout that were fed diets containing with $\mathrm{SO}$ and LO was significantly higher than that in rainbow trout that were fed diets containing FO $(p<0.05)$, and the expression of foxo1 in the LO group was significantly higher than that in the SO group $(p<0.05)$ (Figure $8 \mathrm{~B})$.

A

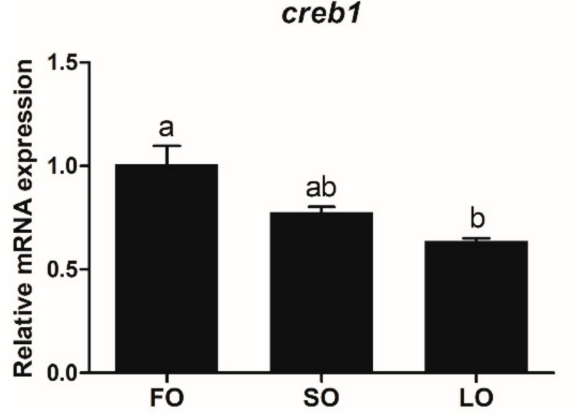

B

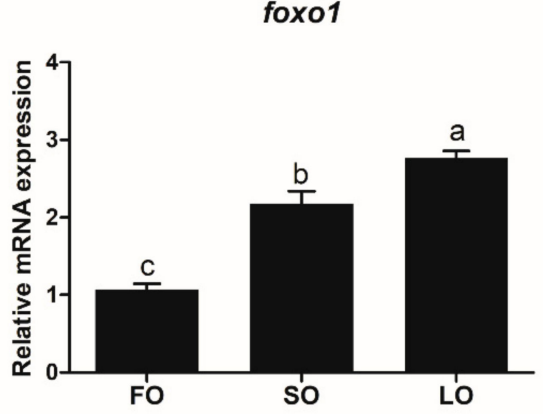

Figure 8. The transcriptional expression of creb1 (A) and foxo1 (B) in the liver of rainbow trout that were fed diets containing fish oil (FO), soybean oil (SO), and linseed oil (LO). The expression in FO was selected as normalization. Data were presented as means \pm S.E.M. $(n=3)$. Means that share the same superscript letter are not significantly different $(p>0.05)$.

\subsubsection{Transcriptional Expression of Creb1 and Foxo1 in Hepatocytes in Response to Insulin}

The transcriptional expression of $c r e b 1$ in hepatocytes treated with insulin for $6 \mathrm{~h}$ showed no significant difference among the four groups $(p>0.05)$. Compared with the control group, the expression of creb1 in hepatocytes of rainbow trout treated with 10 and $100 \mathrm{nM}$ insulin for $8 \mathrm{~h}$ was significantly decreased $(p<0.05)$ (Figure 9A). There were no significant differences between the $6 \mathrm{~h}$ and $8 \mathrm{~h}$ groups (Figure 9A).

Compared with the control group, the transcriptional expression of foxo1 in hepatocytes of rainbow trout treated with 10 and $100 \mathrm{nM}$ insulin for $6 \mathrm{~h}$ was significantly decreased $(p<0.05)$. Furthermore, the transcriptional expression of foxo1 in hepatocytes treated with 1,10, and $100 \mathrm{nM}$ insulin for $8 \mathrm{~h}$ was significantly decreased compared to the control group, and the transcriptional expression of foxo1 in hepatocytes treated with 10 and $100 \mathrm{nM}$ insulin for $6 \mathrm{~h}$ was significantly higher than that in the $8 \mathrm{~h}$ group $(p<0.05)$ (Figure 9B). 
A

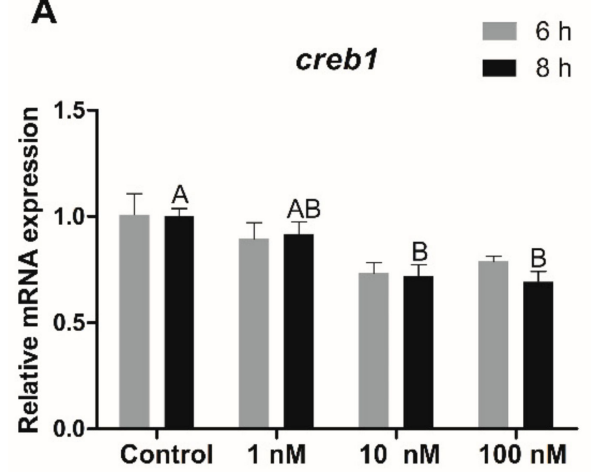

B

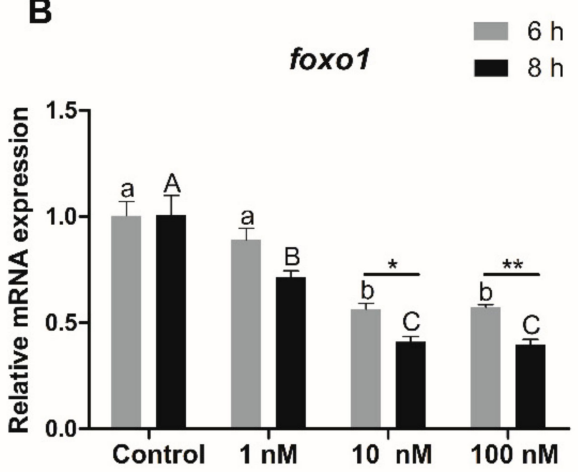

Figure 9. The transcriptional expression of creb1 (A) and foxo1 (B) in hepatocytes of rainbow trout treated with graded amounts of insulin. Control group was selected as normalization. Data were presented as means \pm S.E.M. $(n=3)$. Capital letters show the differences in the $8 \mathrm{~h}$ group. Lowercase letters show the differences in the $6 \mathrm{~h}$ group. Means that share the same superscript letter are not significantly different $(p>0.05) .{ }^{*} p<0.05 ; * * p<0.01$.

\section{Discussion}

As a microsomal enzyme, Elovl6 is specially positioned in the endoplasmic reticulum, which played a crucial role in the elongation of palmitate (PA) or palmitoleate (POA) to stearate or cis-vaccenic acid $[13,14]$. Previous studies in mammals demonstrated that Elovl6 is also involved in inflammatory and metabolic diseases, such as high-fat-diet-induced inflammation and insulin resistance [3,6,15]. However, few studies about Elovl6 in fish have been reported. In the present study, ORF of Elovl6 was cloned from the rainbow trout, which possessed the histidine box (HXXHH) and six membrane-spanning domains of Elovl proteins [16], and showed high identity (87\%) with mammals and teleost. In rainbow trout, the high expression level of elovl 6 was observed in brain and liver tissues, which is consistent with the results in mice [2,4,5] and large yellow croaker [7].

In the present study, the expression of elovl6 in the liver of rainbow trout that were fed diets containing $\mathrm{FO}$ was higher than that in the $\mathrm{SO}$ and $\mathrm{LO}$ groups. Our studies showed that fish oil in diets is richer in PA, POA, EPA, and DHA than soybean oil and linseed oil [7]. PA and POA are the substrates for the synthesis of C18:0 and C18:1n-7 [17]. Hence, the large amount of PA and POA in FO may promote the expression level of elovl6 in rainbow trout to drive the synthesis of fatty acids [18]. A similar effect of PA on elovl6 expression was also observed in large yellow croaker [7]. Furthermore, the transcriptional expression of elovl6 in hepatocytes of rainbow trout treated with EPA and DHA was also higher than that in the ALA and LA groups, which may be another evidence for the high expression level of elovl6 in the FO group. However, studies of large yellow croaker showed that elovl6 expression in SO and LO groups was significantly higher than that in the FO group [7]. The results indicated that the elovl6 of rainbow trout in response to dietary fatty acids is different from large yellow croaker. Rainbow trout, as a freshwater fish, generally has a relatively higher capacity for LC-PUFA synthesis from C18 PUFA (ALA or LA) in comparison to large yellow croaker (marine fish) where such a capacity is normally considerably lower. Hence, ALA and LA were considered as the essential fatty acids for rainbow trout, while the essential fatty acids for large yellow croaker were LC-PUFAs. Thus, the different responses to dietary fatty acids may be due to the difference in the requirements of essential fatty acids and evolutionary position between rainbow trout and large yellow croaker.

Previous studies in mammals have verified the crucial role of elovl6 in insulin resistance $[3,19]$. Mice with a global deficiency for elovl6 could protect against diet-induced insulin resistance [3], and elovl6 specific deletion in the liver of mice ameliorated insulin resistance by reducing hepatic ceramide and protein phosphatase 2A activity [20]. However, studies on the relationship between elovl6 and insulin in fish have not been reported to date. In the present study, the expression of elovl6 in hepatocytes treated with 10 and $100 \mathrm{nM}$ insulin for 6 or $8 \mathrm{~h}$ was significantly increased in rainbow trout. The results demonstrated that the transcriptional expression of elovl6 in rainbow trout could 
be regulated by insulin. Previous studies have shown that insulin could promote the synthesis of lipids by the regulation of transcription factors related to lipid metabolism [21-23]. Thus, it could be speculated that the expression of some transcription factors would be changed when the hepatocytes are treated with the insulin, which may influence the expression of elovl6 in rainbow trout. However, the precise mechanism is still not understood.

To explore the regulatory mechanism of elovl6 expression in response to fatty acids or insulin, dual-luciferase reporter assay has been conducted with the elovl6 promoter and transcription factors plasmids. Previous studies in mammals have reported that elovl6 is consistently regulated by SREBP-1c,2 and PPAR $\gamma[2,5,18,24]$. However, the transcription factor PPAR $\gamma$ showed no regulatory activity on the elovl6 promoter in rainbow trout. In addition, CEBP $\alpha, \mathrm{P} 65, \mathrm{SREBP} 1, \mathrm{SREBP} 2$, and CREB1 could up-regulate the promoter activity of elovl6, and CREB1 showed the largest activation of elovl6 promoter activity in rainbow trout. CREB1 is a key intracellular transcription factor, which played a crucial role in regulating genes of lipid metabolism, such as PPAR $\gamma$, ATGL, and UCP1 [25-28]. The similar activation of CREB1 and SREBP1 on elovl6 has also been observed in large yellow croaker [7]. Furthermore, transcription factors CEBP $\beta, \mathrm{HNF} 1 \alpha$, and $\mathrm{RXR} \alpha$ showed significant activation of the elovl6 promoter in large yellow croaker [7], but no regulatory effects were observed in rainbow trout. The difference in transcriptional regulation of elovl6 by the transcription factors in two fishes may be due to the different requirements of essential fatty acids and evolutionary position. Surprisingly, the FOXO1, which links to insulin signaling and lipid metabolism [29-32], significantly down-regulated the activity of the elovl6 promoter in rainbow trout. Previous studies highlighted the role of FOXO in integrating insulin signaling to hepatic glucose and lipid metabolism [33]. The present study showed the potential regulatory effect of FOXO1 on elovl6 in fish, which may contribute to building up the interaction between the elovl6 and insulin by FOXO1.

Studies on large yellow croaker have demonstrated that the transcription factors would influence the elovl6 expression in response to fatty acids [7], and similar results have also been observed in mammals [18,24]. In the present study, the transcription factor CREB1 showed the most significant activation of elovl6 promoter activity, and FOXO1 greatly down-regulated the promoter activity of elovl6. Thus, CREB1 and FOXO1 were chosen as the main transcription factor to further explain the regulation of elovl6 expression in rainbow trout in response to fatty acids or insulin. The results showed that the expression of creb1 in the liver of rainbow trout that were fed diets containing LO was significantly lower than that in the FO group and the low expression of creb1 may make a decrease of elovl6 expression in the LO group. Furthermore, the expression of foxo1 in the SO and LO groups was significantly higher than that in the FO group, and foxo1 could suppress the expression of elovl6. Thus, the decreased expression of creb1 and increased expression of foxo1 may explain the lower expression of elovl6 in the SO and LO groups than in the FO group. Meanwhile, the creb1 and foxo1 expression in hepatocytes treated with insulin was also analyzed. The significant decrease in the expression of creb1 was only observed in the $8 \mathrm{~h}$ group, but not dramatically. However, the dramatic decrease in expression of foxo1 was observed both in $6 \mathrm{~h}$ and $8 \mathrm{~h}$ group. There is a double down-regulatory effect of elovl6 by creb1 and foxo1 in the $8 \mathrm{~h}$ group, which may explain why the elovl6 expression in hepatocytes treated with 10 and $100 \mathrm{nM}$ insulin for $6 \mathrm{~h}$ was higher than that in the $8 \mathrm{~h}$ group. Thus, the dramatic decrease in expression of foxo1 may mainly explain the increase in elovl6 expression of hepatocytes in response to insulin. Furthermore, previous studies have shown that transcription factors including SREBP1, SREBP2, and CEBP $\alpha$ are also related to fatty acid metabolism and insulin signaling [12,34-41]. We speculated that the expression of srebp1, srebp2, and cebp $\alpha$ may also have effects on the transcriptional expression of elovl6 in response to fatty acids or insulin. However, the speculation should be verified in the future. Overall, the transcription factors CREB1 and FOXO1 might be the potential targets to regulate the transcriptional expression of elovl6 in rainbow trout in response to fatty acids or insulin. These results could help us to further understand the regulatory mechanism of nutrition and hormone in rainbow trout, and aid in the healthy and sustainable wide aquaculture of rainbow trout. 


\section{Conclusions}

In conclusion, the cDNA of elovl6 from rainbow trout was cloned in the present study, which showed the typical features of Elovl proteins. The transcriptional expression of elovl6 in rainbow trout that were fed diets containing FO was higher than that in rainbow trout that were fed diets containing $\mathrm{SO}$ and $\mathrm{LO}$, and the expression of elovl6 was increased with the treatment of insulin. The transcription factor CREB1 could greatly up-regulate the activity of the elovl6 promoter, and FOXO1 greatly down-regulated the activity. The differences in expression of creb1 and foxo1 may contribute to the increase or decrease of elovl6 expression in rainbow trout in response to fatty acids or insulin.

Author Contributions: All authors have read and agree to the published version of the manuscript. Conceptualization, Q.A. and Y.L.; data curation, Y.L. and Y.P.; writing-original draft preparation, Y.L. and Y.P.; writing-review \& editing, Z.Z. and X.X.; supervision, K.M. and Q.A.

Funding: This research was funded by the National Science Fund for Distinguished Young Scholars of China (grant number 31525024), Scientific and Technological Innovation of Blue Granary (Grant no: 2018YFD0900402) and the National Special Support Program for High-Level Personnel Recruitment.

Acknowledgments: We thank Xiang Xu, Zhaoyang Yin and Shengnan Gao for their help in fish rearing.

Conflicts of Interest: The authors declare no conflict of interest.

\section{References}

1. Ameer, F.; Scandiuzzi, L.; Hasnain, S.; Kalbacher, H.; Zaidi, N. De novo lipogenesis in health and disease. Metab. Clin. Exp. 2014, 63, 895-902. [CrossRef]

2. Moon, Y.-A.; Shah, N.A.; Mohapatra, S.; Warrington, J.A.; Horton, J.D. Identification of a mammalian long chain fatty acyl elongase regulated by sterol regulatory element-binding proteins. J. Biol. Chem. 2001, 276, 45358-45366. [CrossRef]

3. Matsuzaka, T.; Shimano, H.; Yahagi, N.; Kato, T.; Atsumi, A.; Yamamoto, T.; Inoue, N.; Ishikawa, M.; Okada, S.; Ishigaki, N.; et al. Crucial role of a long-chain fatty acid elongase, Elovl6, in obesity-induced insulin resistance. Nat. Med. 2007, 13, 1193-1202. [CrossRef]

4. Matsuzaka, T.; Shimano, H. Elovl6: A new player in fatty acid metabolism and insulin sensitivity. J. Mol. Med. 2009, 87, 379-384. [CrossRef]

5. Matsuzaka, T.; Shimano, H.; Yahagi, N.; Yoshikawa, T.; Amemiya-Kudo, M.; Hasty, A.H.; Okazaki, H.; Tamura, Y.; Iizuka, Y.; Ohashi, K.; et al. Cloning and characterization of a mammalian fatty acyl-CoA elongase as a lipogenic enzyme regulated by SREBPs. J. Lipid Res. 2002, 43, 911-920. [PubMed]

6. Matsuzaka, T.; Atsumi, A.; Matsumori, R.; Nie, T.; Shinozaki, H.; Suzuki-Kemuriyama, N.; Kuba, M.; Nakagawa, Y.; Ishii, K.; Shimada, M.; et al. Elovl6 promotes nonalcoholic steatohepatitis. Hepatology 2012, 56, 2199-2208. [CrossRef] [PubMed]

7. Li, Y.; Pang, Y.; Xiang, X.; Du, J.; Mai, K.; Ai, Q. Molecular cloning, characterization, and nutritional regulation of Elovl6 in large yellow croaker (Larimichthys crocea). Int. J. Mol. Sci. 2019, 20, 1801. [CrossRef] [PubMed]

8. Zhao, H.; Matsuzaka, T.; Nakano, Y.; Motomura, K.; Tang, N.; Yokoo, T.; Okajima, Y.; Han, S.-I.; Takeuchi, Y.; Aita, Y.; et al. Elovl6 deficiency improves glycemic control in diabetic $\mathrm{db} / \mathrm{db}$ mice by expanding $\beta$-cell mass and increasing insulin secretory capacity. Diabetes 2017, 66, 1833-1846. [CrossRef]

9. Chen, J.; Cui, Y.; Yan, J.; Jiang, J.; Cao, X.; Gao, J. Molecular characterization of elongase of very long-chain fatty acids 6 (elovl6) genes in Misgurnus anguillicaudatus and their potential roles in adaptation to cold temperature. Gene 2018, 666, 134-144. [CrossRef]

10. Shi, Q.Y.; Yang, Z.G.; Yao, Q.Q.; Cheng, Y.X.; Yang, Q.; Wei, B.H. Full-length cDNA cloning of Elovl6 and its tentative study in Chinese mitten crab (Eriocheir sinensis). J. Fish. China 2016, 40, 844-855. [CrossRef]

11. Livak, K.J.; Schmittgen, T.D. Analysis of relative gene expression data using real-time quantitative PCR and the $2^{-\Delta \Delta C T}$. Methods 2001, 25, 402-408. [CrossRef] [PubMed]

12. Li, S.; Monroig, Ó.; Wang, T.; Yuan, Y.; Carlos, N.J.; Hontoria, F.; Liao, K.; Tocher, D.R.; Mai, K.; Xu, W.; et al. Functional characterization and differential nutritional regulation of putative Elovl5 and Elovl4 elongases in large yellow croaker (Larimichthys crocea). Sci. Rep. 2017, 7, 2303. [CrossRef] [PubMed]

13. Bae, J.S.; Oh, A.R.; Lee, H.J.; Ahn, Y.H.; Cha, J.Y. Hepatic Elovl6 gene expression is regulated by the synergistic action of ChREBP and SREBP-1c. Biochem. Biophys. Res. Commun. 2016, 478, 1060-1066. [CrossRef] [PubMed] 
14. Nakamura, Y.; Matsuzaka, T.; Tahara-Hanaoka, S.; Shibuya, K.; Shimano, H.; Nakahashi-Oda, C.; Shibuya, A. Elovl6 regulates mechanical damage-induced keratinocyte death and skin inflammation. Cell Death Dis. 2018, 9, 1181. [CrossRef] [PubMed]

15. Saito, R.; Matsuzaka, T.; Karasawa, T.; Sekiya, M.; Okada, N.; Igarashi, M.; Matsumori, R.; Ishii, K.; Nakagawa, Y.; Iwasaki, H.; et al. Macrophage Elovl6 deficiency ameliorates foam cell formation and reduces atherosclerosis in low-density lipoprotein receptor-deficient mice. Arterioscler. Thromb. Vasc. Biol. 2011, 31, 1973-1979. [CrossRef] [PubMed]

16. Jakobsson, A.; Westerberg, R.; Jacobsson, A. Fatty acid elongases in mammals: Their regulation and roles in metabolism. Prog. Lipid Res. 2006, 45, 237-249. [CrossRef]

17. Green, C.D.; Ozguden-Akkoc, C.G.; Wang, Y.; Jump, D.B.; Olson, L.K. Role of fatty acid elongases in determination of de novo synthesized monounsaturated fatty acid species. J. Lipid Res. 2010, 51, 1871-1877. [CrossRef]

18. Shi, H.; Wu, M.; Zhu, J.; Zhang, C.; Yao, D.; Luo, J.; Loor, J.J. Fatty acid elongase 6 plays a role in the synthesis of long-chain fatty acids in goat mammary epithelial cells. J. Dairy Sci. 2017, 100, 4987-4995. [CrossRef]

19. Sun, H.; Jiang, T.; Wang, S.; He, B.; Zhang, Y.; Piao, D.; Yu, C.; Wu, N.; Han, P. The effect of LXR $\alpha$, ChREBP and Elovl6 in liver and white adipose tissue on medium- and long-chain fatty acid diet-induced insulin resistance. Diabetes Res. Clin. Pract. 2013, 102, 183-192. [CrossRef]

20. Matsuzaka, T.; Kuba, M.; Koyasu, S.; Yamamoto, Y.; Motomura, K.; Arulmozhiraja, S.; Ohno, H.; Sharma, R.; Shimura, T.; Okajima, Y.; et al. Hepatocyte Elovl6 determines ceramide acyl-chain length and hepatic insulin sensitivity in mice. Hepatology 2019. [CrossRef]

21. Saltiel, A.R.; Kahn, C.R. Insulin signalling and the regulation of glucose and lipid metabolism. Nature 2001, 414, 799-806. [CrossRef]

22. Kim, J.B.; Sarraf, P.; Wright, M.; Yao, K.M.; Mueller, E.; Solanes, G.; Lowell, B.B.; Spiegelman, B.M. Nutritional and insulin regulation of fatty acid synthetase and leptin gene expression through ADD1/SREBP1. J. Clin. Investig. 1998, 101, 1-9. [CrossRef] [PubMed]

23. Foretz, M.; Guichard, C.; Ferré, P.; Foufelle, F. Sterol regulatory element binding protein-1c is a major mediator of insulin action on the hepatic expression of glucokinase and lipogenesis-related genes. Proc. Natl. Acad. Sci. USA 1999, 96, 12737-12742. [CrossRef] [PubMed]

24. Kumadaki, S.; Matsuzaka, T.; Kato, T.; Yahagi, N.; Yamamoto, T.; Okada, S.; Kobayashi, K.; Takahashi, A.; Yatoh, S.; Suzuki, H.; et al. Mouse Elovl6 promoter is an SREBP target. Biochem. Biophys. Res. Commun. 2008, 368, 261-266. [CrossRef]

25. Ortega-Martínez, S. A new perspective on the role of the CREB family of transcription factors in memory consolidation via adult hippocampal neurogenesis. Front. Mol. Neurosci. 2015, 8, 46. [CrossRef] [PubMed]

26. Muraoka, M.; Fukushima, A.; Viengchareun, S.; Lombès, M.; Kishi, F.; Miyauchi, A.; Kanematsu, M.; Doi, J.; Kajimura, J.; Nakai, R.; et al. Involvement of SIK2/TORC2 signaling cascade in the regulation of insulin-induced PGC- $1 \alpha$ and UCP-1 gene expression in brown adipocytes. Am. J. Physiol. Endocrinol. Metab. 2009, 296, E1430-E1439. [CrossRef] [PubMed]

27. Herzig, S.; Hedrick, S.; Morantte, I.; Koo, S.-H.; Galimi, F.; Montminy, M. CREB controls hepatic lipid metabolism through nuclear hormone receptor PPAR- $\gamma$. Nature 2003, 426, 190-193. [CrossRef]

28. Sun, J.; Deng, W.; Gou, N.-N.; Ji, H.; Du, Z.-Y.; Chen, L.-Q. CIDEA and CIDEC are regulated by CREB and are not induced during fasting in grass carp Ctenopharyngodon idella adipocytes. Comp. Biochem. Physiol. B Biochem. Mol. Biol. 2019, 234, 50-57. [CrossRef]

29. Cheng, Z.; Guo, S.; Copps, K.; Dong, X.; Kollipara, R.; Rodgers, J.T.; Depinho, R.A.; Puigserver, P.; White, M.F. FOXO1 integrates insulin signaling with mitochondrial function in the liver. Nat. Med. 2009, 15, 1307-1311. [CrossRef]

30. Zhu, X.; Wu, Y.-B.; Zhou, J.; Kang, D.-M. Upregulation of lncRNA MEG3 promotes hepatic insulin resistance via increasing FOXO1 expression. Biochem. Biophys. Res. Commun. 2016, 469, 319-325. [CrossRef]

31. O-Sullivan, I.; Zhang, W.; Wasserman, D.H.; Liew, C.W.; Liu, J.; Paik, J.; DePinho, R.A.; Stolz, D.B.; Kahn, C.R.; Schwartz, M.W.; et al. FOXO1 integrates direct and indirect effects of insulin on hepatic glucose production and glucose utilization. Nat. Commun. 2015, 6, 7079. [CrossRef] [PubMed] 
32. Mirdamadi, Y.; Thielitz, A.; Wiede, A.; Goihl, A.; Papakonstantinou, E.; Hartig, R.; Zouboulis, C.C.; Reinhold, D.; Simeoni, L.; Bommhardt, U.; et al. Insulin and insulin-like growth factor-1 can modulate the phosphoinositide-3-kinase/Akt/FOXO1 pathway in SZ95 sebocytes in vitro. Mol. Cell. Endocrinol. 2015, 415, 32-44. [CrossRef] [PubMed]

33. Lee, S.; Dong, H.H. FOXO integration of insulin signaling with glucose and lipid metabolism. J. Endocrinol. 2017, 233, R67-R69. [CrossRef] [PubMed]

34. Owen, J.L.; Zhang, Y.; Bae, S.-H.; Farooqi, M.S.; Liang, G.; Hammer, R.E.; Goldstein, J.L.; Brown, M.S. Insulin stimulation of SREBP-1c processing in transgenic rat hepatocytes requires p70 S6-kinase. Proc. Natl. Acad. Sci. USA 2012, 109, 16184-16189. [CrossRef]

35. Tian, J.; Goldstein, J.L.; Brown, M.S. Insulin induction of SREBP-1c in rodent liver requires LXR $\alpha$-C/EBP $\beta$ complex. Proc. Natl. Acad. Sci. USA 2016, 113, 8182-8187. [CrossRef]

36. Botolin, D.; Wang, Y.; Christian, B.; Jump, D.B. Docosahexaneoic acid (22: 6, n-3) regulates rat hepatocyte SREBP-1 nuclear abundance by Erk-and 26S proteasome-dependent pathways. J. Lipid Res. 2006, 47, 181-192. [CrossRef]

37. Boughanem, H.; Cabrera-Mulero, A.; Millán-Gómez, M.; Garrido-Sánchez, L.; Cardona, F.; Tinahones, F.J.; Moreno-Santos, I.; Macías-González, M. Transcriptional analysis of FOXO1, C/EBP- $\alpha$ and PPAR- $\gamma 2$ genes and their association with obesity-related insulin resistance. Genes 2019, 10, 706. [CrossRef]

38. Wu, Y.L.; Peng, X.E.; Wang, D.; Chen, W.N.; Lin, X. Human liver fatty acid binding protein (hFABP1) gene is regulated by liver-enriched transcription factors $H N F 3 \beta$ and C/EBP $\alpha$. Biochimie 2012, 94, 384-392. [CrossRef]

39. Madison, B.B. Srebp2: A master regulator of sterol and fatty acid synthesis. J. Lipid Res. 2016, 57, 333-335. [CrossRef]

40. Suzuki, R.; Lee, K.; Jing, E.; Biddinger, S.B.; McDonald, J.G.; Montine, T.J.; Craft, S.; Kahn, C.R. Diabetes and insulin in regulation of brain cholesterol metabolism. Cell Metab. 2010, 12, 567-579. [CrossRef]

41. Miao, J.; Haas, J.T.; Manthena, P.; Wang, Y.; Zhao, E.; Vaitheesvaran, B.; Kurland, I.J.; Biddinger, S.B. Hepatic insulin receptor deficiency impairs the SREBP-2 response to feeding and statins. J. Lipid Res. 2014, 55, 659-667. [CrossRef] [PubMed] 